\title{
Patient waiting time and satisfaction in GP clinic at a tertiary hospital in Thailand
}

\author{
Apichart Boonma*, Kanchana Sethanan, Sukangkana Talangkun and Teerawat Laonapakul \\ Industrial Engineering Department, Faculty of Engineering, Khon Kaen University, Khon Kaen, Thailand
}

\begin{abstract}
This study aims to investigate patient waiting time in General Practice Clinic and identify the main cause of the problem through patient satisfaction survey. 9,232 Patients were randomly observed and quantitative data such as arrival time and waiting time were recorded. Other 1,200 patients were randomly selected for satisfaction survey which is considered as qualitative data. The result indicated that average waiting time of patients was 175.87 minutes with standard deviation of 68.66 minutes. Waiting time at GP clinic appeared to be the longest with an average of 92.9 minutes and standard deviation of 43.4 minutes. Also, based on the obtained data, patients remained positive until their waiting time exceeded 2 hours.
\end{abstract}

\section{Introduction}

Recently, there has been increasing attention to quality of care in hospitals and healthcare providers around the globe. Deteriorating quality in health services is a major concern in many countries as they are challenged by problems such as the approaching of an aging society, significant rise in healthcare costs, and etc. Patient satisfaction was reported to have an impact on health service quality [1]. Several studies have investigated the factors affecting patient dissatisfaction and addressed that patient waiting time was one of the major causes [2, $3,4,5]$.

Waiting time is the amount of time a patient spent on being seen or treated in a healthcare institution. It is one of the six key quality indexes in US healthcare system and widely used to evaluate performance and efficiency of health services $[6,7]$. Long waiting time does not only slow down the medical treatment, it also increases stress, dissatisfaction, healthcare cost, and may even cause fatality to patients.

Outpatient department (OPD) is where patients are examined and given medications without staying overnight. Among all OPDs, General Practice Clinic (GP) is one of the most crowed departments. It is also the foremost clinic that most patients need to visit. GP doctors typically help patients with different conditions such as acute medical and surgical problems, long-term health problems (e.g. diabetes and asthma), ENT (ear, nose and throat), and dermatology (skin). The OPD patients therefore often experience long waiting time, long length of stay, and dissatisfaction.

The objective of this study is to explore patient waiting time and its cause based on data extracted from patient satisfaction survey at GP clinic of a tertiary hospital in Thailand.

\section{Materials and methods}

The case study hospital is a tertiary hospital and a teaching school for medical students in the Northeast of Thailand. At the time of study, the hospital had about 1,466 beds available for inpatients. Hospital record indicated that average annual outpatient and inpatient visits increased from 718,527 in 2010 to 828,931 in 2013 ( $15 \%$ increment). With this large number of patient visits, waiting time in the hospital has attracted more attention recently.

This study was conducted during Jan - March 2014 at a GP clinic of the case study hospital located in the Northeastern of Thailand. Patients were randomly observed without prior notification. All hospital staffs not involved in the study were not informed about the existence of the study. Observers were placed at main service stations to record important statistics. Two different types of dataset were gathered including quantitative and qualitative data.

The quantitative data consist of arrival, waiting, and leaving time of patients who visited main service stations (triage, medical record, financial support, x-ray, lab test, medical examination, cashier, and pharmacy). Flow chart demonstrating outpatient flow in the hospital is shown in Fig 1. Prior to data collection, sample size was calculated using equation (1).

$$
S=\left[\left(1 / e^{2}\right) z^{2} p(1-p)\right] /\left[1+\left(z^{2} p(1-p) /\left(e^{2} N\right)\right)\right]
$$

Where $S$ is the sampling size, $N$ is population size, $e$ is margin of error, and $z$ is z-score. In this study, $N$ is the average number of patients who daily visit GP clinic which is about 120 patients per day. The prevalence of the factor under study $(p)$ was set to 0.8 . The margin of

\footnotetext{
* Corresponding author: apicbo@kku.ac.th
} 
error and z-score were set to 0.05 and 1.96 respectively. The z-score of 1.96 represents the confident level of $95 \%$. The result suggested that the sample size should be 80.64 or 81 patients.

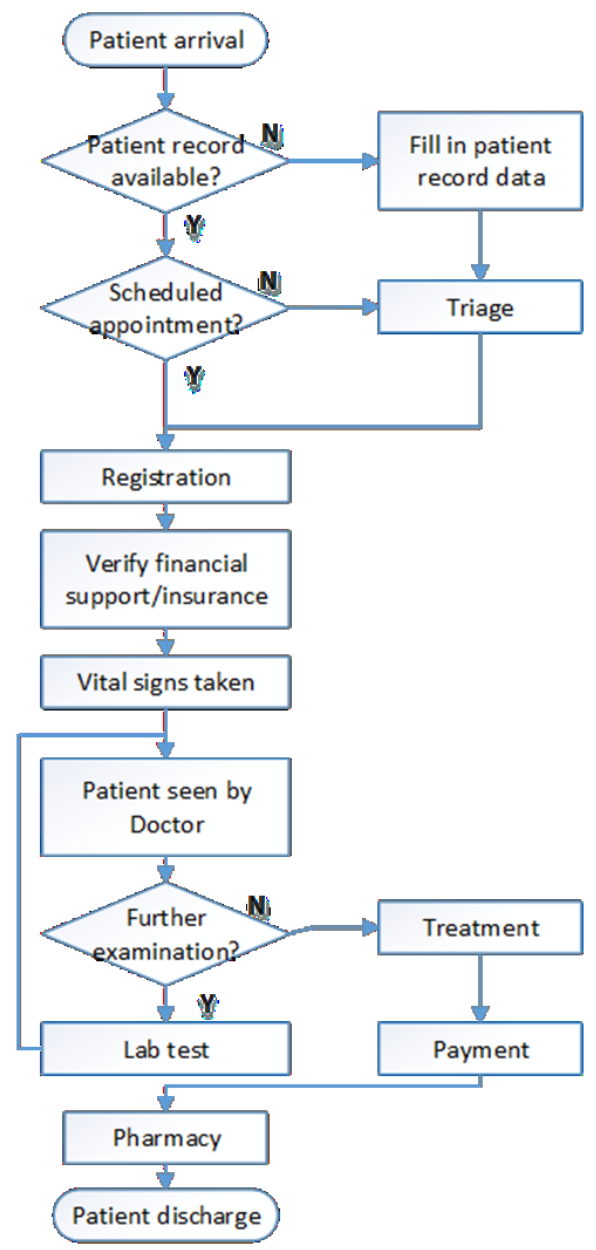

Fig 1. Flow chart illustrating outpatient activities after entering the hospital.

The qualitative data are developed from patient satisfaction survey containing a set of questions. Patients were asked to give their opinion on a scale of 1 to 10 . The survey was conducted at the following service stations; triage, medical record, financial support, x-ray, lab test, medical examination, cashier, and pharmacy. All collected data were analyzed using Analysis ToolPak add-in for Microsoft Excel and Rockwell's Arena Input Analyzer.

\section{Results}

\subsection{Patient arrival}

Based on the observation (Monday - Friday), total number of patients being observed for analyzing arriving pattern was 9,232 patients. Patient arrival times at each station were recorded and then analyzed using Rockwell Arena Input Analyzer with an attempt to identify patient arrival distribution. Based on mean square error (MSE), best fitted distribution can be illustrated as shown in Table 1. The data indicated that patient inter-arrival time was ranging between $0.5-3$ minutes.
Table 1. Best fitted statistical distribution of patient arrival

\begin{tabular}{|c|c|c|}
\hline Section & Expression & MSE \\
\hline Triage & $-0.5+\operatorname{LOGN}(2.76,2.73)$ & 0.007080 \\
\hline Registration & $-0.5+\operatorname{LOGN}(2.04,2.15)$ & 0.004021 \\
\hline Insurance & $-0.5+\operatorname{LOGN}(1.28,1.17)$ & 0.003281 \\
\hline Laboratory & $-0.5+\operatorname{LOGN}(1.46,1.31)$ & 0.007911 \\
\hline Xray & $-0.001+\operatorname{EXPO}(2.78)$ & 0.000289 \\
\hline GP clinic & $-0.001+\operatorname{WEIB}(1.78,0.469)$ & 0.002852 \\
\hline Cashier & $-0.5+\operatorname{ERLA}(0.709,2)$ & 0.015499 \\
\hline Pharmacy & $-0.5+\operatorname{LOGN}(0.989,0.784)$ & 0.003613 \\
\hline
\end{tabular}

Moreover, the pattern of patient arrival shown in Fig 2 indicates the time each service station was busy. The busiest time for Triage, Medical Record/Insurance, Xray, GP, Cashier, and Pharmacy were 7.00-8.00, 8.00$9.00,9.00-11.00,9.00-12.00$, and 10.00-12.00 respectively. After 10.00, Pharmacy and Cashier had largest number of patient visits. Since doctors often start working at OPD around 9.00, patients who have been diagnosed and given medication or prescription arrive at the stations around some time after that. Laboratory, on the contrary, was very busy early morning (6.00 10.00). This is due to the fact that patients are required to have laboratory tests (e.g. blood, urine sampling, etc) before they can be seen by doctors.

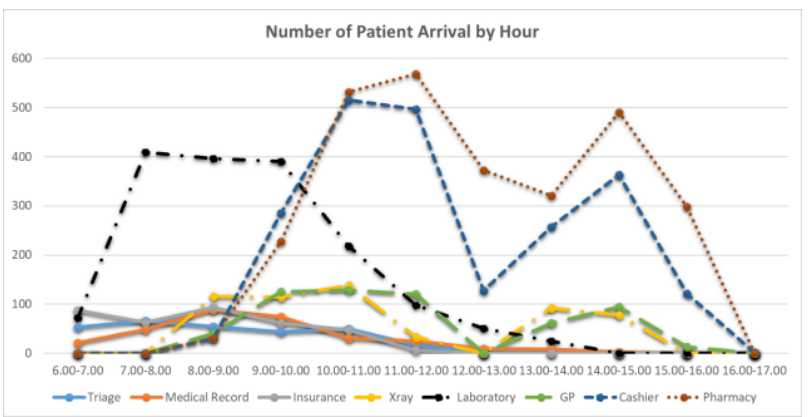

Fig 2. Patient arrival at each service station by hour

\subsection{Waiting time}

Waiting time or the time patients have to wait idly before they can get serviced is one of the most favorite key performance index to evaluate the performance of healthcare units. In our study, waiting time was determined as the time a patient checked in at a service station and waited until he/she received service or treatment.

When patients arrive at the hospital, they need to go to triage first for initial interview. There were 2-3 nurses stationed in triage. A total of 284 patients were observed at triage. The data showed that most patients $(85.92 \%)$ received service as soon as they arrived. The remaining $(14.08 \%)$, however, waited up to 13 minutes. The average waiting time was about 3.53 minutes with standard deviation of 2.95 . 
Next, patients need to complete registration process. There was an average of 3.5 staffs posed at this station and total 309 patients were monitored. One hundred and fifty-nine patients $(51.46 \%)$ waited less than 5 minutes. Seventy-four patients $(23.95 \%)$ waited between 5 and 10 minutes. Forty-nine patients $(15.86 \%)$ waited for 10 to 20 minutes. Twenty-one (6.8\%) and six patients $(1.94 \%)$ waited for 20-30 minutes and more than 30 minutes respectively. The average waiting time of 6.7 minutes was obtained with standard deviation of 7.61.

Prior to being seen by physician, patients need to inform hospital staffs regarding their health insurance and payment method. There were 4-7 hospital staffs providing support at this station (5.5 staffs on average). Total 81 out of 358 observed patients $(22.63 \%)$ waited between 5 and 10 minutes. Nine patients $(2.51 \%)$ waited longer than 10 minutes. The average waiting time at this process was 3.65 minutes with standard deviation of 2.47 .

In case blood sample or other lab tests are required, patients need to visit clinical laboratory and go through blood sampling procedure. An average of 4.04 staffs providing service at this station. Total of 2,661 patients were observed and their waiting time are listed as shown by the following 1 ) less than 5 minutes $-1,803$ patients $(67.76 \%), 2) 5-10$ minutes - 294 patients $(11.05 \%), 3)$ 10-20 minutes - 227 patients $(8.53 \%)$, 4) 20-30 minutes - 122 patients $(4.58 \%), 5)$ longer than 40 minutes -71 patients $(2.67 \%)$. The average waiting time was 7.56 minutes with standard deviation of 10.91 .

At Xray, an average of 2.79 rooms were available with 2 staffs per room. Total of 1,212 patients were observed and their waiting time are listed as follows 1) less than 5 minutes - 375 patients $(30.94 \%)$, 2) $5-10$ minutes -49 patients $(4.04 \%)$, 3) 10-20 minutes -85 patients $(7.01 \%)$, 4) 20-30 minutes - 193 patients (15.92\%), 5) 30-40 minutes - 216 patients $(17.82 \%), 6)$ 40-50 minutes -120 patients $(9.90 \%)$, 7) 50-60 minutes -123 patients $(10.15 \%)$, and 8 ) more than 60 minutes 51 patients $(4.21 \%)$. The average waiting time was 25.63 minutes with standard deviation of 22.25 .

Services at GP clinic were divided into 2 stages, 1) Patient Interview and 2) Medical Examination. Patients arrived at GP clinic were first interviewed by a nurse and then they waited for their turn to get examined by a doctor. For patient interview where nurses asked patients for their information, number of patients and their waiting time are listed as follows 1) less than 5 minutes - 68 patients, 2) 5-10 minutes - 20 patients $(3.83 \%), 3)$ 10-20 minutes - 29 patients $(5.56 \%), 4)$ 20-30 minutes 42 patients $(8.05 \%)$, 5) 30-40 minutes - 52 patients $(9.96 \%), 6)$ 40-50 minutes - 40 patients $(7.66 \%), 7) 50$ 60 minutes -43 patients $(8.24 \%)$, 8) 60-120 minutes 153 patients $(29.31 \%)$, and 9) longer than 120 minutes 75 patients $(14.37 \%)$. The average waiting time at patient interview was 63.70 minutes with standard deviation of 51.92 .

For medical examination, there were 2-13 doctors providing service depending on the day of the week and the time of the day (5.74 doctors on average). Number of patients and their waiting time are listed as follows, 1) less than 5 minutes - 66 patients $(11.40 \%)$, 2) 5-10 minutes - 69 patients (11.92\%), 3) 10-20 minutes - 126 patients $(21.76 \%)$, 4) $20-30$ minutes - 92 patients $(15.89 \%), 5) 30-40$ minutes -80 patients $(13.82 \%), 6)$ $40-50$ minutes -50 patients $(8.64 \%)$, 7) 50-60 minutes 44 patients $(7.60 \%)$, 8) 60-120 minutes -47 patients $(7.60 \%)$ and 9) more than 120 minutes - 5 patients $(0.86 \%)$. The average waiting time at medical examination was 29.20 minutes with standard deviation of 24.27. Considering both patient interview and medical examination as single process, the average waiting time became 92.9 minutes with standard deviation of 43.40 .

After consulting with a doctor, patients make payment at Cashier. There were 2-5 staffs available at this station (3.37 staffs on average). 1981 out of 2039 patients $(97.16 \%)$ received service within 5 minutes. Fifty-two patients $(2.55 \%)$ had to wait between 5 to 10 minutes before they could get serviced. Only 6 patients $(0.29 \%)$ received service after waiting longer than 10 minutes. The average waiting time was about 1 minute with standard deviation of 1.54 .

Lastly, patients can pick up medication at Pharmacy. There were 2-7 staffs posed at this station (average of 4.58). Number of patients and their waiting times are listed as follows 1) less than 5 minutes - 42 patients $(1.26 \%), 2) 5-10$ minutes - 212 patients $(6.34 \%), 3) 10$ 20 minutes -864 patients $(25.85 \%)$, 4) $20-30$ minutes 719 patients $(21.51 \%), 5) 30-40$ minutes -533 patients (21.51\%), 6) 40-50 minutes - 347 patients $(10.38 \%), 7)$ 50-60 minutes -229 patients $(6.85 \%), 8) 60-120$ minutes -341 patients $(10.20 \%)$, and 9) longer than 120 minutes - 56 patients $(1.68 \%)$. The average waiting time at Pharmacy was 34.90 minutes with standard deviation of 27.21.

\subsection{Patient satisfaction}

Patient satisfaction is considered qualitative data in our study. Total number of patients who participated in the survey was 1,200 patients. The questionnaires were evenly distributed to the patients receiving services at four different departments including Medical Record, GP clinic, Cashier, and Pharmacy. There were 10 questions in the questionnaire, but, there was only a single question directly relating to patient waiting time. The results of satisfaction survey can be shown by follows.

At Registration, patients participating in the survey consisted of 122 males and 178 females. Patients rated how fast they received service as 6.03 on a scale of 10 . Most patients thought waiting time was the result of a large number of patients and insufficient staffs.

At GP clinic, total 140 males and 160 females were interviewed. Their response to service speed was 4.73 on a scale of 10 . They also mentioned that in their opinion the delay was caused by a great number of early registered patients.

Three hundred patients consisting of 141 males and 159 females at Cashier agreed to take part in the survey. Their response to the service at this station was 5.57 out 
of 10. One of the main cause was insufficient staffs and service desks.

Lastly, at Pharmacy, total 110 males and 190 females gave a score of 6.30 out of 10 for the services they received. The main cause of delay was reported similar to that found at GP clinic.

\section{Discussion}

Based on the data shown in Fig 3, the longest waiting time was observed at GP clinic (92.9 minutes). Pharmacy comes second (34.9 minutes), and the third and fourth are medical exam (29.2 minutes) and Xray (25.63 minutes) respectively. Early patient arrival and limited number of hospital staffs are probably the primary causes of the problem. The similar finding was found in another secondary hospital in Thailand where the bottleneck processes are patient interview and medical exam.

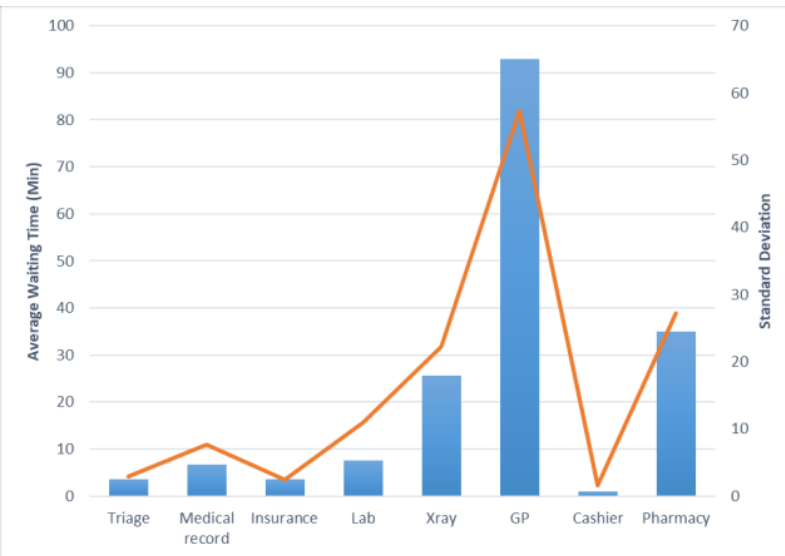

Fig 3. Average waiting time at each service station

The data also indicated total waiting time that patients required in addition to processing time to complete all services at the case study hospital was approximately 175.87 minutes with standard deviation of 68.66 on the average. This amount of time falls within the range of time that patients in most developing countries spent in outpatient department (2-4 hours). It is also in close agreement to total waiting time observed in Michigan (USA), Nigeria, and Trinidad and Tobago where 188,173 , and 160 minutes were recorded [8, 9, 10]. Shorter waiting time was reported elsewhere such as Atlanta (USA - 60 minutes), and Toronto (Canada 126.79 minutes) [1]. These data are shown in Fig 4.

Considering the satisfaction survey, the minimum satisfaction score of 4.73 was discovered at GP clinic. It is the only station that received a score less than 5 and thus its satisfaction level is considered as "below average". This is probably due to the fact that about $50 \%$ of patients had to wait at least 2 hours before they could be seen by a doctor. This is four times longer than the recommended waiting time of the Institute of Medicine [11].

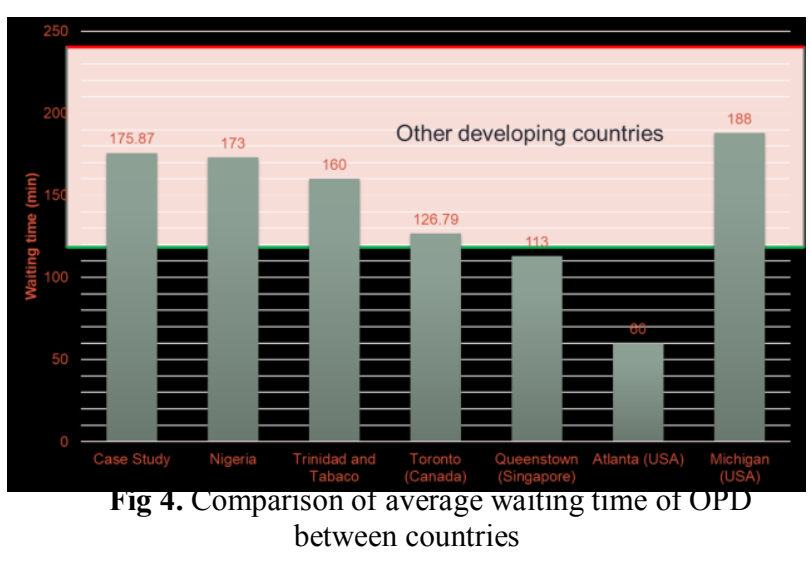

Also, from the questionnaires, the primary causes of long waiting time perceived by patients include large number of patients receiving service, insufficient staffs, and early arrival of patients. As Thailand is changing into aging society, an increase in patients needing health service has become more common. Moreover, since the case study hospital is a famous teaching hospital in the region, many patients requiring advance diagnosis and treatment are transferred here. In Thailand, although the ratio of doctor and nurse to patient of 2.28 per 1000 population is higher than that recommended by World Health Organization of 1 per 1000 population [12], allocating manpower to cope with incoming patients is still a challenge. Many patients still believe that early bird registration would minimize waiting time and they therefore come to the hospital extremely early. This does not only prolong the average waiting time of every patient, but it also applies pressure to hospital staff and eventually decrease their performance and efficiency. To make the problem worse, most patients in the case study hospital are walk-in patients who come to the hospital without making prior arrangements. This is different from hospital in developed countries where patients are required to make appointment before their arrival.

\section{Conclusion}

Our findings indicated that patient waiting time of 175.87 minutes or 2.93 hours at the case study hospital is in good agreement with other hospital in other developing countries. Also, patient dissatisfaction was discovered once patients need to wait longer than 2 hours. To prepare for increasing number of patients due to aging society, there is a need for GP clinic to develop necessary strategies in order to reducing waiting time. Staff allocation should be planned according to patient incoming pattern, while patients should be familiar and comfortable with making appointment before arriving at the hospital. Moreover, efficient appointment system should be used in order to properly distributing patients across the hospital service hours. Further research should focus on measuring related factors contributing to waiting time in more details, develop, and evaluate possible solutions. 


\section{Acknowledgements}

This research was partially funded by Faculty of Engineering, Khon Kaen University. The author would also like to thank the managements and staffs of the case study hospital for their support, cooperation and suggestion.

\section{References}

1. K. Syed, D. Parente, S. Johnson, J. Davies. Factors determining wait-time and patient satisfaction at post-operative orthopaedic follow-up. OJMP 2(1), 47-53 (2013).

2. X. M. Huang. Patient attitude towards waiting in an outpatient clinic and its applications. Health Serv Manage Res 7(1), 2-8 (1994).

3. L.P. Fenny, T. Smith, J. Dwyer. Client flow analysis: A practical management technique for outpatient clinic settings. Int J Qual Health Care 6, 179-186 (1994).

4. K. C. Chung, J. B. Hamil, H. M. Kim, M. R. Walters, E. G. Wilkins. Predictors of patient satisfaction in an outpatient plastic surgery clinic. Ann Plast Surg 42(1), 56-60 (1999).

5. J. Levesque, E. R. Bogoch, B. Cooney, B. Johnson, J. G. Wright. Improving patient satisfaction with time spent in an orthopedic outpatient clinic. Can J Surg 43(6), 431-436 (2000)

6. R. J. Maxwell. Quality assessment in health. Br Med J (Clin Res Ed) 288(6428), 1470-1472 (1984).

7. Committee on Quality of Health Care in America; Institute of Medicine, Crossing the Quality Chasm: A New Health System for the $21^{\text {st }}$ Century (National Academies Press, 2001).

8. L. M. dos Santos, G. Stewart, N. M. Rosenberg. Pediatric emergency department walk-outs. Pediatr Emerg Care 10(2), 76-78 (1994).

9. A. N. Ofilli, C. E. Ofowye. What patients want: A content analysis of key qualities that influence patient satisfaction. J Med Pract Manage 22, 255261 (2007).

10. A. N. Ofilli, C. E. Ofowye. Patient's assessment of efficiency of services at a teaching hospital in a developing country. Ann Afr Med 4, 150-153 (2005).

11. M. S. O’Malley, S. W. Fletcher, R. H. Fletcher, J. A. Earp, J Ambul Care Manage 6, 20-27 (1983).

12. M. O. Oche, H. Adamu. Determinants of patient waiting time in the General Outpatient Department of a tertiary health institution in North Western Nigeria. Ann Med Health Sci Res 3(4), 588-592 (2013). 